\title{
Spontaneous Heparin-Induced Thrombocytopenia Following Orthopaedic Surgery: Case Report and Review of the Literature
}

\author{
Malcolm R. DeBaun'1, Michael A. Spinner², Michael D. Genualdi³, Caroline Berube ${ }^{2}$, \\ Derek F. Amanatullah'1 \\ ${ }^{1}$ Department of Orthopaedic Surgery, Stanford University School of Medicine, Stanford, CA, USA \\ ${ }^{2}$ Division of Hematology, Department of Medicine, Stanford University School of Medicine, Stanford, CA, USA \\ ${ }^{3}$ Department of Medicine, Stanford University School of Medicine, Stanford, CA, USA \\ Email: mdebaun@stanford.edu
}

How to cite this paper: DeBaun, M.R., Spinner, M.A., Genualdi, M.D., Berube, C. and Amanatullah, D.F. (2017) Spontaneous Heparin-Induced Thrombocytopenia Following Orthopaedic Surgery: Case Report and Review of the Literature. Open Journal of Orthopedics, 7, 400-407.

https://doi.org/10.4236/ojo.2017.712042

Received: October 21, 2017

Accepted: December 2, 2017

Published: December 5, 2017

Copyright $\odot 2017$ by authors and Scientific Research Publishing Inc. This work is licensed under the Creative Commons Attribution International License (CC BY 4.0).

http://creativecommons.org/licenses/by/4.0/

\begin{abstract}
Background: Spontaneous heparin induced thrombocytopenia (HIT) is a medical phenomenon whereby patients develop clinical and laboratory features of HIT without prior exposure to heparin. Aim: We present a patient who underwent bilateral total knee replacement complicated by spontaneous HIT causing bilateral adrenal hemorrhage and deep vein thrombosis. Our patient had no prior history of heparin exposure. We reconcile our patient's presentation with other case reports of spontaneous HIT in patients who had orthopaedic surgery. Conclusion: Spontaneous HIT is a rare but potentially devastating complication associated with total knee arthroplasty.
\end{abstract}

\section{Keywords}

Spontaneous Heparin Induced Thrombocytopenia, HIT, Arthroplasty, Total Knee Arthroplasty, TKA

\section{Introduction}

Spontaneous HIT is an increasingly recognized phenomenon whereby patients develop clinical and laboratory features of HIT, including thrombocytopenia, thrombosis, and positive serologic markers, without prior exposure to unfractionated or low molecular weight heparin [1]. In traditional HIT, heparin and platelet factor 4 (PF4) combine to form a neoepitope immunoglobulin complex capable of activating platelets and ultimately leading to a highly prothrombotic 
condition [2] [3] [4]. Clinical suspicion for HIT should arise in patients who experience a significant drop in their platelet count typically between 5 and 10 days after heparin exposure, especially in the setting of a new thrombotic event. Serologic testing then can identify the circulating IgG/PF4/heparin complexes, and the diagnosis of HIT is subsequently confirmed with a functional platelet activation assay such as the serotonin release assay or aggregometry. If the patient has not had prior exposure to heparin despite positive serologic testing, the diagnosis of spontaneous HIT is made. Clinically, patients with HIT can present with venous and/or arterial thromboses, including deep vein thrombosis (DVT), pulmonary embolism (PE), ischemic stroke, myocardial infarction, acute limb ischemia, skin necrosis, acute mesenteric ischemia, or adrenal hemorrhage secondary to adrenal vein thrombosis leading to venous outflow obstruction.

The first case of spontaneous HIT following orthopaedic surgery was described by Jay and Warkentin [5]. This was a fatal complication in a patient who underwent total knee arthroplasty (TKA) and subsequently developed clinical and serologic manifestations of HIT on postoperative day 9 without prior exposure to heparin. Since this inaugural report, there have been at least 10 other described diagnoses of spontaneous HIT in patients after orthopaedic surgery with the vast majority of presentations occurring after TKA. Simultaneous bilateral surgery, intraoperative tourniquet use, and postoperative utilization of dynamic mechanical thromboprophylaxis have been theorized as risk factors [6] [7] [8]. The mechanism of spontaneous HIT following orthopedic surgery is thought to involve the release of glycosaminoglycans such as chondroitin sulfate, a polyanion which structurally resembles heparin and is capable of forming platelet-activating complexes with PF4 [9] [10].

We report a case of spontaneous HIT in a patient who underwent simultaneous bilateral TKA and subsequently presented with severe symptomatic adrenal insufficiency secondary to bilateral adrenal hemorrhage as well as new thrombotic disease. Of note, our patient was also ingesting a preoperative supplement containing chondroitin sulfate, serving as another possible inciting factor for the development of spontaneous HIT [9]. Herein, we summarize our patient's presentation and reconcile with a comprehensive review of the literature on spontaneous HIT following orthopaedic surgery in an effort to bring awareness to this rare but potentially fatal complication. Consent was obtained from the patient to participate in this case study.

\section{Case Report}

A 68-year-old male with a history of hyperlipidemia and chronic bilateral knee pain secondary to osteoarthritis underwent uncomplicated bilateral TKA. On postoperative day 1 , he was started on prophylactic dosing rivaroxaban $10 \mathrm{mg}$ once daily for DVT prevention. He had no prior exposure to unfractionated or low molecular weight heparin. The patient progressed well with physical therapy until postoperative day 10 where he was noted to have developed generalized le- 
thargy and weakness. Laboratory analysis obtained at this time was notable for new anemia (hemoglobin $6.6 \mathrm{~g} / \mathrm{dL}$, preoperative hemoglobin $14.5 \mathrm{~g} / \mathrm{dL}$ ), thrombocytopenia (platelet count $46,000 / \mu \mathrm{L}$, preoperative platelet count $205,000 / \mu \mathrm{L}$ ), and hyponatremia (sodium $118 \mathrm{mmol} / \mathrm{L}$ ), in addition to revealing a urinary tract infection (UTI). He was given intravenous fluids and started on nitrofurantoin without symptomatic improvement.

On postoperative day 13 , he was admitted to our institution with progressive generalized weakness and lethargy. He was started on intravenous ceftriaxone for the urinary tract infection and received intravenous fluids for presumed hypovolemic hyponatremia. He received one unit of packed red blood cells. Rivaroxaban was discontinued given concern for acute bleeding in the setting of persistent thrombocytopenia. Further work up revealed a low serum cortisol level of $1.3 \mu \mathrm{g} / \mathrm{dL}$ (normal $>2.0 \mu \mathrm{g} / \mathrm{dL}$ ), which failed to respond to cosyntropin stimulation (post-stimulation cortisol $1.0 \mu \mathrm{g} / \mathrm{dL}$ ) and an elevated serum adrenocorticotropic hormone (ACTH) level at $128 \mathrm{pg} / \mathrm{mL}$ (normal $10-60 \mathrm{pg} / \mathrm{mL}$ ) consistent with a primary adrenal insufficiency. A CT scan of the abdomen and pelvis demonstrated bilateral adrenal hemorrhage with periadrenal stranding (Figure 1(a)). The patient was then started on intravenous hydrocortisone with significant improvement in his mental status and hyponatremia.
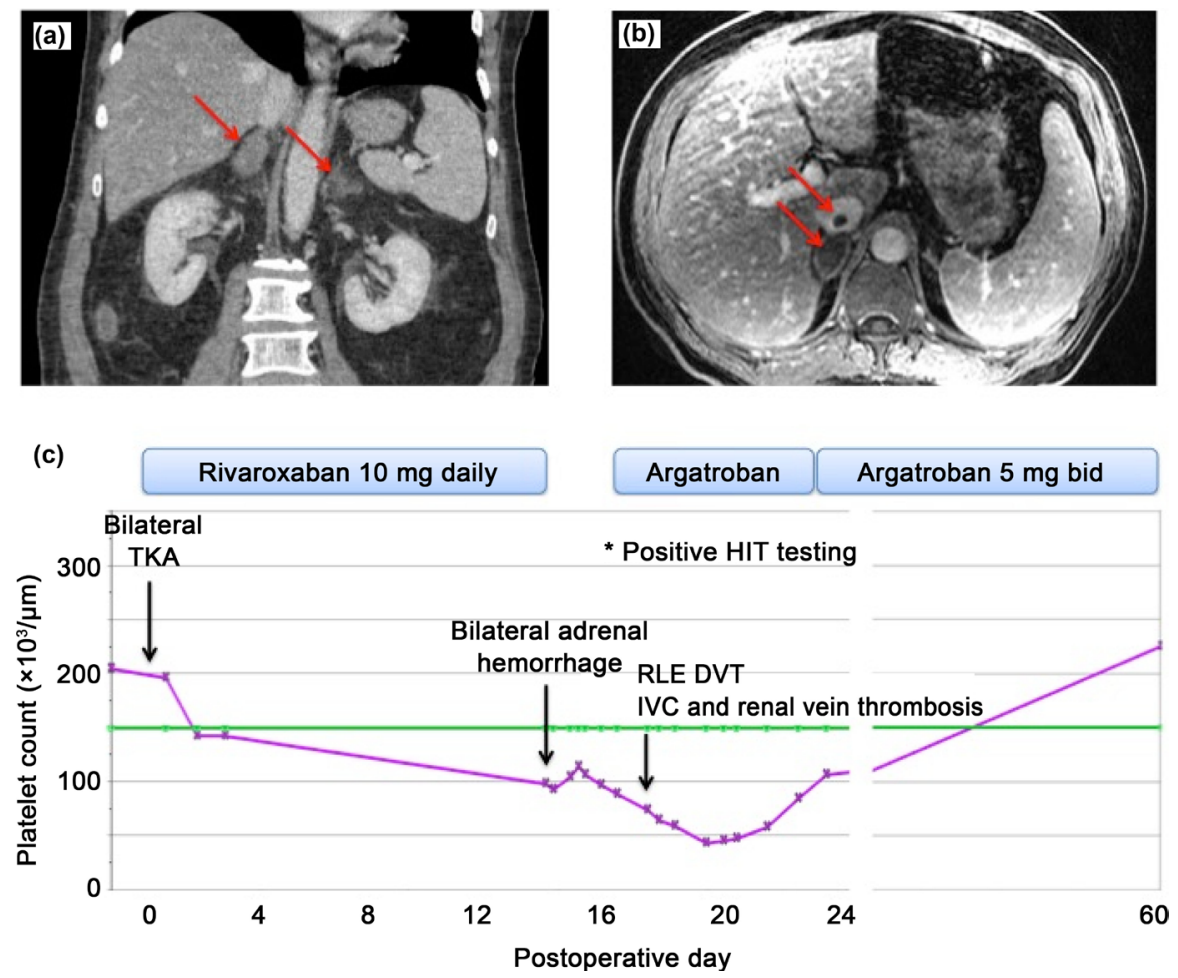

Figure 1. Radiographic findings and clinical course. (a) CT scan of the abdomen and pelvis. Arrows indicate enlargement of the bilateral adrenal glands with high attenuation values consistent with hemorrhage. (b) MRI of the abdomen. Arrows indicate thrombus within the inferior vena cava (top arrow) and hematoma in the right adrenal gland (bottom arrow). (c) Temporal summary of the patient's clinical course, platelet count trend, and anticoagulation management. Green line indicates the lower limit of normal platelet count. 
Concurrently, the patient's anemia and thrombocytopenia were further evaluated with the assistance of the hematology consult service. Comprehensive work up for other sources of anemia was negative, in addition to negative blood cultures for infection. Following the diagnosis of bilateral adrenal hemorrhage, additional laboratory testing was obtained including antiphospholipid syndrome serologies, which were negative, and serologic testing for HIT. Despite absence of prior heparin exposure, serologic testing for HIT was markedly abnormal (PF4/IgG optical density units 3.037, normal $<0.40$ ) and a subsequent positive functional platelet assay confirmed the diagnosis of HIT. Screening bilateral lower extremity ultrasounds revealed an occlusive thrombosis in the right common femoral vein and partially occlusive thrombi in the right femoral, popliteal, and posterior tibial veins. An adrenal protocol MRI of the abdomen also incidentally showed a small amount of thrombus in the intrahepatic inferior vena cava and left renal vein (Figure 1(b)). The patient was started on on intravenous argatroban and his platelet count improved from a nadir of $43,000 / \mu \mathrm{L}$ on postoperative day 19 to $106,000 / \mu \mathrm{L}$ on post-operative day 23 . He was transitioned to oral anticoagulation on therapeutic dose apixaban $5 \mathrm{mg}$ twice daily and discharged on postoperative day 24 . At a follow up clinic visit 1 month later, he was recovering well without any further thrombotic events with normalization of his platelet count to $225,000 / \mu \mathrm{L}$ and hemoglobin to $13.2 \mathrm{~g} / \mathrm{dL}$. The patient's temporal clinical course including platelet count, thrombotic events, and anticoagulation management are summarized in Figure 1(c).

\section{Discussion}

We present a patient who underwent bilateral TKA complicated by HIT without prior heparin exposure. Postoperatively, the patient regressed with physical therapy and was noted to have developed anemia, thrombocytopenia and hyponatremia at the skilled nursing facility. After readmission to the hospital, further work up revealed bilateral adrenal hemorrhage with associated adrenal insufficiency and venous thrombotic disease. Ultimately, the diagnosis of spontaneous HIT was made after confirmation with serologic testing. The patient was then appropriately treated with therapeutic anticoagulation and corticosteroids and made a full recovery.

We identified 16 published cases of spontaneous HIT occurring after orthopaedic surgery, with all but one case occurring after TKA (Table 1). All patients had at least a $50 \%$ postoperative reduction in platelet count, venous and/or arterial thromboses, high-titer elevated PF4 antibodies detected between postoperative day 9 to 18 , and a positive functional assay confirming the diagnosis of HIT. Routine postoperative thromboprophylaxis with warfarin, aspirin, fondaparinux, or apixaban was administered in all reported cases, indicating that a prophylactic dosing of anticoagulation is not sufficient to prevent thrombosis in the setting of HIT.

Ten of the 16 patients with spontaneous HIT following orthopaedic surgery 
Table 1. Published cases of spontaneous HIT and fondaparinux-associated HIT following orthopedic surgery.

\begin{tabular}{|c|c|c|c|c|c|c|c|c|}
\hline Publication, year & $\begin{array}{l}\text { Patient } \\
\text { age/sex }\end{array}$ & $\begin{array}{l}\text { Orthopedic } \\
\text { surgery }\end{array}$ & $\begin{array}{c}\text { DVT } \\
\text { prophylaxis }\end{array}$ & $\begin{array}{l}\text { POD at } \\
\text { diagnosis }\end{array}$ & $\begin{array}{l}\text { Platelet } \\
\text { nadir }\end{array}$ & Thrombotic events & $\begin{array}{l}\text { PF4 IgG } \\
\text { OD }\end{array}$ & $\begin{array}{l}\text { Functional } \\
\text { assay }\end{array}$ \\
\hline $\begin{array}{l}\text { Warkentin et al., } \\
\qquad 2007\end{array}$ & $48 \mathrm{~F}$ & Bilateral TKA & Fondaparinux & 10 & 39,000 & $\begin{array}{l}\text { Bilateral adrenal hemorrhage } \\
\text { Right lower extremity DVT }\end{array}$ & 1.87 & + SRA \\
\hline Jay et al., 2008 & $69 \mathrm{~F}$ & Right TKA & Warfarin & 9 & 11,000 & $\begin{array}{l}\text { Bilateral adrenal hemorrhage } \\
\text { Right lower extremity DVT } \\
\text { Digital infarcts }\end{array}$ & 1.89 & + SRA \\
\hline Pruthi et al., 2009 & $61 \mathrm{~F}$ & Right TKA & Warfarin & 10 & 15,000 & $\begin{array}{l}\text { Bilateral adrenal hemorrhage } \\
\text { Bilateral DVTs/PEs }\end{array}$ & 2.30 & + SRA \\
\hline Salem et al., 2010 & $67 \mathrm{M}$ & Left TKA & Fondaparinux & 9 & 35,000 & $\begin{array}{l}\text { Bilateral DVTs } \\
\text { Ischemic CVA }\end{array}$ & 2.803 & ND \\
\hline Mallik et al., 2011 & $60 \mathrm{M}$ & Bilateral TKA & Warfarin & 10 & 15,000 & Bilateral DVTs & 3.0 & + SRA \\
\hline Purch et ol 2012 & $63 \mathrm{M}$ & Right TKA & Fondaparinux & 18 & 50,000 & $\begin{array}{l}\mathrm{DVT} / \mathrm{PE} \\
\text { IVC thrombosis }\end{array}$ & 2.807 & + SRA \\
\hline Durch el al., 2012 & $63 \mathrm{M}$ & Right TKA & Fondaparinux & 12 & 38,000 & $\begin{array}{l}\text { Iliac artery thrombosis } \\
\text { Splenic and left renal infarcts }\end{array}$ & 3.081 & + SRA \\
\hline \multirow{4}{*}{ Ketha et al., 2013} & \multirow{4}{*}{$\begin{array}{l}61 \mathrm{~F} \\
60 \mathrm{~F} \\
65 \mathrm{M} \\
71 \mathrm{~F}\end{array}$} & \multirow{4}{*}{$\begin{array}{l}\text { TKA } \\
\text { TKA } \\
\text { TKA } \\
\text { TKA }\end{array}$} & Warfarin & $9-15$ & 15,000 & $\begin{array}{l}\text { Bilateral adrenal hemorrhage } \\
\text { DVT }\end{array}$ & Elevated & + SRA \\
\hline & & & Warfarin & $9-15$ & 81,000 & $\begin{array}{l}\text { Bilateral adrenal hemorrhage } \\
\text { DVT }\end{array}$ & Elevated & + SRA \\
\hline & & & Warfarin & $9-15$ & 115,000 & $\begin{array}{l}\text { Bilateral adrenal hemorrhage } \\
\text { DVT }\end{array}$ & Elevated & + SRA \\
\hline & & & Warfarin & $9-15$ & 20,000 & $\begin{array}{l}\text { Bilateral adrenal hemorrhage } \\
\text { DVT }\end{array}$ & Elevated & + SRA \\
\hline $\begin{array}{l}\text { Warkentin et al., } \\
\qquad 2014\end{array}$ & $54 \mathrm{~F}$ & $\begin{array}{l}\text { Shoulder } \\
\text { arthroplasty }\end{array}$ & None & 15 & 37,000 & $\begin{array}{l}\text { Right lower extremity DVT } \\
\text { Ischemic CVA }\end{array}$ & 2.89 & + SRA \\
\hline $\begin{array}{l}\text { Warkentin et al., } \\
\quad 2015\end{array}$ & ND & TKA & Apixaban & 13 & 37,000 & $\begin{array}{l}\text { Bilateral adrenal hemorrhage } \\
\text { DVT/PE } \\
\text { IVC thrombosis }\end{array}$ & 3.02 & + SRA \\
\hline Elshoury et al., 2015 & $48 \mathrm{~F}$ & Left TKA & Aspirin & 10 & 15,000 & $\begin{array}{l}\text { Bilateral adrenal hemorrhage } \\
\text { Bilateral PEs }\end{array}$ & Elevated & + SRA \\
\hline Baker et al., 2017 & $72 \mathrm{~F}$ & Left TKA & Aspirin & 12 & 25,000 & Bilateral DVTs/PEs & 2.97 & + SRA \\
\hline DeBaun et al., 2017 & $68 \mathrm{M}$ & Bilateral TKA & Rivaroxaban & 16 & 43,000 & $\begin{array}{l}\text { Bilateral adrenal hemorrhage } \\
\text { Right lower extremity DVT } \\
\text { IVC and renal vein thrombosis }\end{array}$ & 3.037 & +HITIA \\
\hline
\end{tabular}

$\mathrm{CVA}=$ cerebrovascular accident, DVT $=$ deep vein thrombosis, HIT $=$ heparin-induced thrombocytopenia, HITIA $=$ heparin-induced thrombocytopenia impedance aggregometry, IVC = inferior vena cava, ND = no data, OD = optical density, PE = pulmonary embolism, PF4 = platelet factor 4 , POD = post-operative day, $\mathrm{SRA}=$ serotonin release assay, $\mathrm{TKA}=$ total knee arthroplasty.

presented with bilateral adrenal hemorrhage (63\%), which is a well-described manifestation of HIT [11] [12] [13] [14]. Paradoxically, adrenal hemorrhage is caused by venous outflow obstruction from adrenal vein thrombosis [15]. The resultant adrenal insufficiency can be life-threatening leading to hemodynamic collapse if unrecognized. Symptoms are often vague and non-specific such as fatigue, generalized weakness, nausea, or abdominal pain. Clinically, patients may 
have hypotension unresponsive to resuscitation as well as metabolic abnormalities including hyponatremia and hyperkalemia [16] [17]. Prompt recognition and initiation of corticosteroid therapy is critical. After making the diagnosis of HIT, patients should be started on intravenous therapy with a non-heparin anticoagulant such as argatroban as well as strict avoidance of any heparin-containing products. We recommend continuing intravenous anticoagulation until the platelet count improves, ideally above $100,000 / \mu \mathrm{L}$, and then transitioning to warfarin or a direct oral anticoagulant such as apixaban, rivaroxaban, or dabigatran for at least 3 to 6 months for the management of the acute thrombotic event.

It is notable that nearly all cases of spontaneous HIT following orthopaedic surgery (94\%) occurred after TKA and not other joint replacement surgery. The release of heparin-like molecules such as chondroitin sulfate from within the knee joint into the systemic circulation has been proposed as the mechanism for the development of platelet-activating PF4 complexes following TKA [6]. Interestingly our patient also ingested oral supplements containing chondroitin sulfate pre-operatively. Although there is laboratory evidence that PF4 can form complexes with hypersulfated chondroitin sulfate [9], it is unclear whether oral supplementation with chondroitin sulfate may lead to the formation of systemic platelet-activating PF4 complexes.

In a prospective study of over 2000 patients who underwent TKA or hip arthroplasty, dynamic mechanical prophylaxis was an independent risk factor for the development of anti-PF4/heparin antibody formation even in the absence of prior heparin exposure [7]. The authors speculate that the mechanical stimulation and subsequent tissue damage associated with dynamic mechanical thromboprophylaxis induces anti-PF4/heparin antibodies through the release of polyanions such as glycosaminoglycans or nucleic acids [7] [18]. Alternatively, local tissue ischemia secondary to tourniquet use and the subsequent release of these active polyanions is another proposed mechanism accounting for the disproportionate prevalence of spontaneous HIT seen after TKA in comparison to total hip arthroplasty [7] [8]. Of note, we limit tourniquet use to only during the cementation process when performing TKA making this mechanism less likely in this case and overall.

The temporal course of events and improvement after treatment with argatroban supports the diagnosis of spontaneous HIT in our patient. Nonetheless, our patient had a concomitant urinary tract infection at the time of his presentation that was treated initially with nitrofurantoin and eventually ceftriaxone upon admission. There are described cases of spontaneous HIT associated with gram-negative bacteremia [19]. This mechanism is possible yet unlikely to be relevant in our case, given gram negative bacteria are commonly associated with urinary tract infection. Urine culture results were negative in our patient. Ceftriaxone is a known cause of thrombocytopenia but not applicable given the timing of patient's clinical deterioration was prior to its administration. Anecdotally, presence of antiphospholipid antibodies, which were negative in our pa- 
tient's serological work up, is associated with a spontaneous prothrombotic disorder that resembles HIT [20].

\section{Conclusion}

We describe a new case of spontaneous HIT after bilateral TKA and summarize all other previously published cases of this phenomenon following orthopaedic surgery. Orthopaedic surgeons, particularly those who routinely perform TKAs, should be aware of this potentially devastating postoperative complication.

\section{References}

[1] Warkentin, T.E., Makris, M., Jay, R.M. and Kelton, J.G. (2008) A Spontaneous Prothrombotic Disorder Resembling Heparin-Induced Thrombocytopenia. American Journal of Medicine, 121, 632-636. https://doi.org/10.1016/j.amjmed.2008.03.012

[2] Horsewood, P., Warkentin, T.E., Hayward, C. and Kelton, J. (1996) The Epitope Specificity of Heparin-Induced Thrombocytopenia. British Journal of Haematology, 95, 161-167. https://doi.org/10.1046/j.1365-2141.1996.d01-1876.x

[3] Kelton, J.G., Sheridan, D., Santos, A., et al. (1988) Heparin-Induced Thrombocytopenia: Laboratory Studies. Blood, 72, 925-930.

http://www.ncbi.nlm.nih.gov/pubmed/12535302

[4] Warkentin, T.E., Levine, M.N., Hirsh, J., et al. (1995) Heparin-Induced Thrombocytopenia in Patients Treated with Low-Molecular-Weight Heparin or Unfractionated Heparin. The New England Journal of Medicine, 332, 1330-1335. https://doi.org/10.1056/NEJM199505183322003

[5] Jay, R.M. and Warkentin, T.E. (2008) Fatal Heparin-Induced Thrombocytopenia (HIT) during Warfarin Thromboprophylaxis Following Orthopedic Surgery: Another Example of "Spontaneous" HIT? Journal of Thrombosis and Haemostasis, 6, 1598-1600. https://doi.org/10.1111/j.1538-7836.2008.03040.x

[6] Mallik, A., Carlson, K.B. and DeSancho, M.T. (2011) A Patient with "Spontaneous" Heparin-Induced Thrombocytopenia and Thrombosis after Undergoing Knee Replacement. Blood Coagulation \& Fibrinolysis, 22, 73-75.

https://doi.org/10.1097/MBC.0b013e328340ff11

[7] Bito, S., Miyata, S., Migita, K., et al. (2016) Mechanical Prophylaxis Is a Heparin-Independent Risk for Anti-Platelet Factor 4/Heparin Antibody Formation after Orthopedic Surgery. Blood, 127, 1036-1043. https://doi.org/10.1182/blood-2015-06-651620

[8] Warkentin, T.E., Cook, R.J., Marder, V.J. and Greinacher, A. (2010) Anti-PF4/Heparin Antibody Formation Postorthopedic Surgery Thromboprophylaxis: The Role of non-drug risk factors and Evidence for a Stoichiometry-Based Model of Immunization. Journal of Thrombosis and Haemostasis, 8, 504-512.

https://doi.org/10.1111/j.1538-7836.2009.03735.x

[9] Jaax, M.E., Krauel, K., Marschall, T., et al. (2013) Properties of Platelet Factor 4 Complex Formation with Nucleic Acids and Aptamers Alters the Antigenic Properties of Platelet Factor 4. Journal of Thrombosis and Haemostasis, 122, 272-281.

[10] Warkentin, T.E., Basciano, P.A., Knopman, J. and Bernstein, R.A. (2014) Spontaneous Heparin-Induced Thrombocytopenia Syndrome : 2 New Cases and a Proposal for Defining This Disorder. Blood, 123, 3651-3654.

https://doi.org/10.1182/blood-2014-01-549741 
[11] Warkentin, T.E., Safyan, E.L. and Linkins, L.-A. (2015) Heparin-Induced Thrombocytopenia Presenting as Bilateral Adrenal Hemorrhages. The New England Journal of Medicine, 372, 492-494. https://doi.org/10.1056/NEJMc1414161

[12] Ketha, S., Smithedajkul, P., Vella, A., Pruthi, R., Wysokinski, W. and McBane, R. (2013) Adrenal Haemorrhage due to Heparin-Induced Thrombocytopenia. Journal of Thrombosis and Haemostasis, 109, 669-675. https://doi.org/10.1160/TH12-11-0865

[13] Elshoury, A., Khedr, M., Abousayed, M.M. and Mehdi, S. (2015) Spontaneous Heparin-Induced Thrombocytopenia Presenting as Bilateral Adrenal Hemorrhages and Pulmonary Embolism after Total Knee Arthroplasty. Arthroplast Today, 1, 69-71. https://doi.org/10.1016/j.artd.2015.07.003

[14] Rosenberger, L., Smith, P., Sawyer, R., Hanks, J., Adams, R. and Hedrick, T. (2011) Bilateral Adrenal Hemorrhage: The Unrecognized Cause of Hemodynamic Collapse Associated with Heparin-Induced Thrombocytopenia. Critical Care Medicine, 39, 833. https://doi.org/10.1097/CCM.0b013e318206d0eb

[15] Fox, B. (1976) Venous Infarction of the Adrenal Glands. The Journal of Pathology, 119, 65-89. https://doi.org/10.1002/path.1711190202

[16] Picolos, M.K., Nooka, A., Davis, A.B., Raval, B. and Orlander, P.R. (2007) Bilateral Adrenal Hemorrhage: An Overlooked Cause of Hypotension. The Journal of Emergency Medicine, 32, 167-169. https://doi.org/10.1016/j.jemermed.2006.05.046

[17] Rao, R.H., Vagnucci, A.H. and Amico, J.A. (1989) Bilateral Massive Adrenal Hemorrhage: Early Recognition and Treatment. Annals of Internal Medicine, 110, 227-235. https://doi.org/10.7326/0003-4819-110-3-227

[18] Rauova, L., Zhai, L., Kowalska, M.A., Arepally, G.M., Cines, D.B. and Poncz, M. (2006) Role of Platelet Surface PF4 Antigenic Complexes in Heparin-Induced Thrombocytopenia Pathogenesis: Diagnostic and Therapeutic Implications. Blood, 107, 2346-2353. https://doi.org/10.1182/blood-2005-08-3122

[19] Pongas, G., Dasgupta, S.K. and Thiagarajan, P. (2013) Antiplatelet Factor 4/Heparin Antibodies in Patients with Gram Negative Bacteremia. Thrombosis Research, 132, 217-220. https://doi.org/10.1016/j.thromres.2013.06.013

[20] Warkentin, T.E., Makris, M., Jay, R.M. and Kelton, J.G. (2008) A Spontaneous Prothrombotic Disorder Resembling Heparin-Induced Thrombocytopenia. American Journal of Medicine, 121, 632. https://doi.org/10.1016/j.amjmed.2008.03.012 